\title{
Double Periodicity in Be Stars *
}

\author{
D. Clarke and P.A. McGale \\ University Observatory, Acre Road / Maryhill Road, \\ GLASGOW G20 OTL, Scotland U.K.
}

Summary: Photometric periodicities with two unequal maxima and minima reported for Be stars are shown to result from a fundamental and overtone associated with rotation of an atmospheric bulge. It is the form of the scattering function of the free electron that produces the double periodicity; the stellar geometry co-latitude of the bulge and inclination of the stellar rotational pole - controls the amplitudes of the two components. The form of the light curves is consistent with the oblique rotator model.

The Double Periodicity Light Curve Many Be stars display short-term photometric and polarimetric variability. Some suggest that the fluctuations have a stochastic nature with no identifiable stable periodicities. Others display periodicities which are sometimes elusive to identify, perhaps because of variations in the amplitude or because good fits to the variability can be achieved both with a given period or with its harmonic.

Attention has been drawn by Harmanec (1984) to stars with light curves exhibiting two maxima and minima in one apparent period, which, in the absence of radial velocity variations, rules out a possible (eclipsing) binary interpretation in preference to stellar rotation. Harmanec favours that such stars are magnetic oblique rotators, but it is not clear whether he promotes the idea that the complete light curve is caused by the progression across the projected stellar disc of the two patches associated with the opposite poles. More recently Balona and Engelbrecht (1986) have recorded several Be stars displaying the two unequal maxima/minima which they ascribe to star spots.

With a view to identifying the nature of polarimetric variability in Be stars, Clarke and McGale (1986 a,b,c) have investigated a model involving the effect of globules, bulges or enhancements in the number of free electrons in what otherwise might be considered as an axisymmetric extended atmosphere. By allowing the bulge to be in co-rotation with the star, it was demonstrated that the scattering from electrons in the globule provided two simultaneous polarimetric periods, one matching the stellar rotation, the other equal to its first harmonic, the amplitudes depending on the co-latitude, $\theta$, of the bulge and the stellar inclination, 1 . Despite the simplicity of the model involving a point source with the globule being taken as a singularity, the developed analytical procedures allowed the rotation period $\left(0 .{ }^{\mathrm{d}} 992\right)$ for $X$ Per, also giving $i=50^{\circ}$ and $\theta \sim 20^{\circ}$, indicating that the star is an oblique rotator.

* Read by P. Harmanec 
The same model also predicts that a co-rotating bulge will emulate the light curves described above and that it is the simultaneous presence of the fundamental and the harmonic which gives rise to the two unequal maxima/minima. By taking the intensity parameter from the Stokes vector describing the scattered radiation from the bulge, the variable part of the light curve is represented by:

$$
I(t) \propto\left(\tau_{0}(t) / 2\right)\left(\sin ^{2} \theta \sin ^{2} i \cos 2 \omega t-\sin 2 \theta \sin 2 i \cos \omega t\right)
$$

where $\tau_{0}$ is the optical depth of the bulge and $\omega$ the rotational frequency. Thus the strength of the fundamental and the harmonic components depend on the values of $\theta$ and $i$, there being no periodic variation if either $\theta$ or $i$ equal zero, but stochastic changes would still be apparent if $\tau_{0}$ fluctuates; the amplitudes of the periodic variations would also be variable if $\tau_{0}$ changes. Two similar globules diametrically opposed is equivalent to doubling $\tau_{0}$.

The double periodicity is best seen when the fundamental and the harmonic are both significantly present. The figure depicts the curve for the fundamental/harmonic amplitude ratio 1:3 for comparison with the star Ahmed 88 ( $c f$. Balona and Engelbrecht (1986)). A feature noted in several stars is the separation of subsequent minima not being exactly 0.5 . This effect is inherently contained in the above equation. The departure from 0.5 depends on the relative strength of the fundamental to harmonic; for example, at $1: 1$, the separation of the minima are approximately 0.58 and 0.42 .

Decoupling the parameters $i$ and $\theta$ from photometry alone is difficult as the equation above shows that they are interchangable. However polarimetry allows the separation to be made and it is suggested that any Be star which exhibits "double periodicity" is an ideal candidate for a polarimetric programme. It may also be noted that for certain geometries it might be the harmonic rather than the fundamental that dominates the photometry.

The combination of the geometry of the oblique rotator and the scattering function of the free electron provides the basic mechanism to explain some of the unusual light curves of Be Stars.
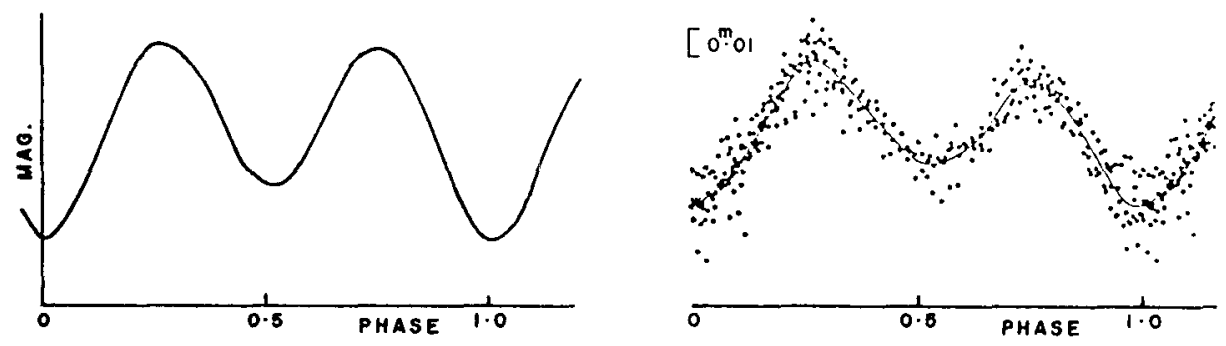

\section{References:}

Balona, L.A., Engelbrecht, C.A.: 1986 M. N. Roy. Astr. Soc. 219, 131 Clarke, D., McGale, P.A.: 1986a In Press - Astron. Astrophys. Clarke, D., McGale, P.A.: 1986b Submitted - Astron. Astrophys. Clarke, D., McGale, P.A.: 1986c Submitted - Astron. Astrophys. Harmanec, P.: 1984 Bull. Astron. Inst. Czechosl. 35, 193 
DISCUSSION FOLLOWING CLARKE AND McGALE (Paper read by Harmanec)

Henrichs:

Would you comment on the $22 \mathrm{~h} x$-ray period found by $\mathrm{N}$. White some 10 years ago?

Harmanec:

Clarke and McGale pay attention to all possible causes of producing spurious periodicity and they believe the nearly one-day period they found in the polarimetric data is real and thus supports the $x$-ray period to be equal or similar to the rotation period of the optical star of X Per - perhaps a somewhat puzzling result! 